\title{
Ongoing theta oscillations predict encoding of subjective memory type
}

Florence I. Kleberg* ${ }^{1}$, Keiichi Kitajo ${ }^{1,2}$, Masahiro Kawasaki ${ }^{2,3}$, Yoko Yamaguchi ${ }^{1}$

1) RIKEN Brain Science Institute,

Saitama, Wako-shi, Hirosawa 2-1

Japan

2) RIKEN BSI-Toyota Collaboration Center, Saitama, Wako-shi, Hirosawa 2-1

Japan

3) Graduate School of Systems and Information Engineering, University of Tsukuba, Ibaraki, Tsukuba-shi, Tennodai 1-1-1

Japan

kleberg@brain.riken.jp*

kkitajo@brain.riken.jp

kawasaki@iit.tsukuba.ac.jp

yokoy@brain.riken.jp

*Corresponding author

Present address: RIKEN Brain Science Institute

Saitama, Wako-shi, Hirosawa 2-1 Japan

phone: 048-462-1111 ext. 7463

fax: 048-467-6899 


\begin{abstract}
Recent human electro-encephalography (EEG) studies show that ongoing brain states support successful encoding of human memory, including recognition. However it is not known whether ongoing cortical activity qualitatively determines different memory types at encoding. In this study, using a Remember/Know procedure, we measured the EEG oscillations that emerge before and during the encoding of abstract visual stimuli in episodic and non-episodic memory, focusing on the theta $(2-8 \mathrm{~Hz})$ and alpha (9-12 $\mathrm{Hz}$ ) oscillation range. We found that enhanced prestimulus theta oscillations precede episodic memory encoding, compared to non-episodic encoding. The prestimulus difference appeared at frontal and temporal sites. Furthermore, the theta enhancement reappeared after stimulus onset. Enhanced upper alpha oscillations suggested increased working memory processing in the case of episodic memory. Finally, the pre- and post-stimulus theta and alpha amplitudes showed different correlation patterns for episodic and non-episodic encoding. Our results are the first to suggest that encoding of episodic memory depends on preparatory processing in the form of frontal and temporal theta oscillations.
\end{abstract}

\title{
Introduction
}

A growing body of evidence suggests that ongoing cortical activity affects our performance in a variety of cognitive tasks (Linkenkaer-Hansen et al., 2004; Hanslmayr et al., 2007; Boly et al., 2007; Van Dijk et al., 2008; Mathewson et al., 2009; Busch et al., 2009; Hipp et al., 2011, Hamm et al., 2012). Ongoing activity before learning, or “encoding” of a stimulus, can even predict learning success (Otten et al., 2006;

Guderian et al., 2009; Fell et al., 2011, Yoo et al., 2012). For instance, fluctuating Blood-Oxygen Level Dependent (BOLD) activity in the parahippocampal cortex predicts whether a stimulus will be subsequently encoded (Yoo et al., 2012). Prestimulus electro-encephalograpy (EEG) also allows predictions of whether a stimulus will be successfully encoded or not, with increased theta oscillations (3-8 Hz) typically preceding successful trials (Guderian et al., 2009; Fell et al., 2011). Although these studies address the question of encoding success or failure, recognizing the stimulus successfully may include awareness of the stimulus context, or not (Mandler, 1980; Jacoby et al., 1991). For instance, a stimulus can be successfully recognized with awareness of its episodic context (episodic memory), or without 
context, but with knowledge of it having been encountered before (non-episodic memory). Episodic and non-episodic memory are therefore different in subjective memory, the qualitative experience of memory, which can be tested either by reporting on one's own retrieval experience (called “metamemory"), or by explicitly reporting a contextual detail that appeared at learning. With such techniques, numerous studies have indicated that episodic versus non-episodic memory encoding and retrieval may be based on different neural processes. For instance, Event-Related Potentials (ERPs) are more positive during encoding of items that are later retrieved with their context, compared to without context (Duarte et al., 2004). ERPs are also more positive during encoding of episodic, compared to non-episodic, items (Voss and Paller, 2009a). Evidence from functional magnetic resonance imaging (fMRI) studies also supports a dissociation in the neural substrate, both during encoding (Davachi et al., 2003; Bowles et al., 2007; Kirwan et al., 2008; de Vanssay-Maigne et al., 2011; Song et al., 2011) and retrieval of stimuli (Wheeler and Buckner, 2004; Yonelinas et al., 2005; de Vanssay-Maigne et al., 2011; Diana et al., 2007). Moreover, frontal scalp-recorded EEG oscillations in the theta range are enhanced during encoding of stimuli with context, compared to without context (Summerfield and Mangels, 2005).

If ongoing activity predicts the successful encoding of memory before the stimulus has appeared (e.g. Otten et al., 2006; Guderian et al., 2009; Fell et al., 2011, Yoo et al., 2012), then does does the prestimulus cortical state before learning influence not only whether we recall, but also how we recall? If so, it would mean that there exist favourable brain states for episode-learning and for single-item-learning, knowledge of which could greatly aid study or learning purposes.

In this study, we address the question of whether the ongoing cortical state determines stimulus encoding with or without context awareness. Participants used self-report of episodic and non-episodic memory, using a recognition memory paradigm known as the "Remember/Know" procedure. In this task, one either recognizes the stimulus with episodic awareness of the context ("Remember", episodic memory) or without this awareness ("Know", non-episodic memory; Tulving, 1985).

We recorded EEG oscillations before and during encoding of the stimuli, analyzed EEG, and performed a post-hoc analysis on a correlation between pre- and post-stimulus 
effects. The focus of our EEG analysis was in the theta $(2-8 \mathrm{~Hz})$ and alpha $(9-12 \mathrm{~Hz})$ range oscillations, as both have been shown to play a prominent role in ongoing predictive activities (Guderian et al., 2009; Fell et al., 2011; Mathewson et al., 2009; Busch et al., 2009) as well as memory processing in general (Winson, 1978; Lisman and Idiart, 1995; Sarnthein et al., 1998; Tesche and Karhu, 2000; Raghavachari et al., 2001; Sauseng et al., 2005a; Osipova et al., 2006; Babiloni et al., 2009; Rutishauser et al., 2010; Friese et al., 2013).

\section{Materials and Methods}

\section{Participants}

Fourteen (four female) healthy, right-handed native Japanese speakers, aged between 20 and 25 years, with normal or corrected-to-normal vision, participated in the experiment after providing written informed consent, according to the RIKEN Ethics Committee. Data from two participants was excluded from the final EEG analyses due to excessive contamination of the recordings through movement-related artifacts, and data from one additional participant was excluded for providing insufficient 'Remember' responses, leaving eleven participants for data analysis. None of the participants had any history of neurological disease.

\section{Design and procedure}

The experimental procedure was a modification of a two-alternative forced-choice (2AFC) paradigm by Voss and Paller (2009b), a recognition memory task encompassing encoding (learning) and retrieval (recall) of visual stimuli, adapted for electrophysiological recordings.

Each participant performed one practice block and 16 experiment blocks. A block consisted of an encoding phase, an arithmetic task phase, and a retrieval phase (Fig. 1A). Per block, 10 stimuli were encoded and retrieved, resulting in 160 trials per participant. At the start of each block, participants were briefly reminded of experiment instructions. During the encoding phase, participants were instructed to fixate the center of the screen, and to limit eye blinks to the start of the inter-stimulus intervals where possible, in order to prevent eye-movement artifact during the relevant EEG recordings. Twelve different kaleidoscopic images were shown in alternation with a fixation cross in the center of the screen. Images lasted $2 \mathrm{~s}$, and fixation crosses $4-4.5 \mathrm{~s}$ (multiples of $50 \mathrm{~ms}$ as jitter). 
The first and the last of the twelve images served as "buffer images" that accounted for primacy and recency effects in memory tasks (“Serial Position Effect”: Deese and Kaufman, 1957). After the encoding phase, participants were prompted to perform an arithmetic task, which served as a distraction between encoding and retrieval, to prevent active rehearsal of stimulus information. Participants selected the correct response from three choices to an arithmetic problem involving subtractions and additions, and received feedback immediately on whether their choice was correct. In the retrieval phase, a brief sequence with two kaleidoscopic images was presented. One image was repeated from the encoding phase ("target stimulus"), while the other was new ("foil"; see stimuli to see how foils were paired up with target stimuli), either preceding it or following it. Target stimuli and foils were not shown simultaneously, as viewing them so would result in EEG artifacts due to eye movements. Participants indicated the temporal position of the target stimulus, by answering "first" or "second", and received no feedback about whether it was correct. A subsequent screen asked participants for the subjective basis of their choice: "Don’t Know", “Know”, or "Remember". Don’t Know if they did not know which image was the target image and had to guess, Know if they knew which image was the target image but did not recollect seeing it in the encoding phase, and Remember if they did recollect the experience of seeing the target image during the encoding phase. Participants were tested only once for each target stimulus. After being tested on all 10 non-buffer stimuli from the encoding phase, participants were informed of the start of the next block.

Stimulus order was randomized throughout, except for the specific pairing of foil and target stimulus during retrieval, which was fixed. All target stimuli, foils and buffer stimuli were unique per block, and each block had its unique three-color scheme for stimuli. Blocks were counterbalanced (pseudorandomized) across participants, and separated by self-paced breaks. All experiments were conducted in a dimly lit room.

\section{Stimuli}

Stimuli were randomly generated, abstract kaleidoscopic images, containing three colors, as inspired by a paradigm by Voss and Paller (2009a, 2009b), that were presented on a black background with a PC and a CRT monitor (Eizo Flexscan GeForce T966) with a refresh rate of $120 \mathrm{~Hz}$, and subtended a visual angle of $4.5 \mathrm{deg}$. Stimuli were presented using Presentation software (Neurobehavioral Systems, Albany, CA) 
and created with custom-written MATLAB code (The Mathworks).

The motivation for using abstract kaleidoscopic stimuli instead of words was to emphasize visual memory and prevent rehearsal of verbal information concerning stimuli. In order to create a degree of visual similarity between the target stimulus and the foil that were paired for testing recognition memory, the foil was derived from the target stimulus by taking the points from the target stimulus figure drawing, shifting them $15 \mathrm{deg}$. up or down (each with a probability of 50\%), and redrawing the line between them. This was performed independently for all three color layers for each foil.

\section{EEG recordings}

During encoding and retrieval, scalp EEG was recorded with $60(\mathrm{Ag} / \mathrm{AgCl})$ cap embedded electrodes (from hereon referred to as channels), which were arranged by the International 10-10 system. The midfrontoanterior (AFz) channel was used to ground the signal. Other additional channels included horizontal and vertical EOG as well as earlobe-reference recordings. The EEG and EOG were recorded with reference to the left earlobe recording, and referenced offline to the right earlobe recording. EEG was amplified with NeuroScan Synamps2 equipment (Neuroscan, EI Paso, Texas, USA). After subtracting half of the right earlobe recording, EEG was filtered offline with a FIR bandpass filter, from EEGLab software (Delorme and Makeig, 2004; Institute for Neural Computation, University of California, San Diego, CA, USA), leaving a frequency range $2 \mathrm{~Hz}-100 \mathrm{~Hz}$ at a $500 \mathrm{~Hz}$ sampling frequency.

\section{EEG processing}

After recording, continuous EEG data was broken up into epochs, including only data surrounding the onset of the stimulus-to-be-remembered in the encoding phase. This lead to $5000 \mathrm{~ms}$ long "raw epochs", that encompassed $1500 \mathrm{~ms}$ prestimulus (before stimulus onset) and $2500 \mathrm{~ms}$ post-stimulus (after stimulus onset) EEG. Ocular artifacts in each raw epoch were rejected with a Blind-Source Separation (BSS) method called Second-Order Blind Identification (SOBI). SOBI was performed with the ICA toolbox in EEGLab software (Delorme and Makeig, 2004) in MATLAB. Artifacts were rejected based on correlations between components of the scalp EEG and components of the horizontal and vertical EOG; components with a correlation of 0.3 or over were removed, and remaining components were regressed back to EEG data. Additional raw 
epochs containing artifacts of over $150 \mu \mathrm{V}$ or below $-150 \mu \mathrm{V}$ were rejected with EEGLab. The numbers of remaining epochs after rejection are shown in Table 1. Current source density estimation, a spherical spline surface Laplacian method, was used to minimize the influence of volume conduction and to accentuate local rather than global changes (Perrin et al., 1989; Kayser and Tenke, 2006). We set the order of the spline at $\mathrm{m}=4$ and the number of iterations at $\mathrm{n}=50$.

In order to find the different frequency components contributing to the EEG, we applied a Finite Impulse Response (FIR) filter to the raw epochs for frequencies between 2 to 45 Hz. The analytic signal was then created with the Hilbert Transform method, where the real part of the oscillating signal is derived from EEG data, and the imaginary part is constructed with the Hilbert operator (equation (1)).

$$
\xi(t)=f(t)+i f(t)=A(t) e^{i \phi(t)}
$$

Where $\mathrm{xi}(\mathrm{t})$ is the time-frequency transformed data, $\mathrm{f}(\mathrm{t})$ is the real part of the raw signal, $\mathrm{f} \sim(\mathrm{t})$ is the Hilbert operator, $\mathrm{A}(\mathrm{t})$ is the amplitude of the raw signal, and phi $(\mathrm{t})$ the phase of the raw signal.

\section{Statistical analysis of EEG data}

For subsequent analysis, only $1000 \mathrm{~ms}$ prestimulus and $1000 \mathrm{~ms}$ post-stimulus data from the raw epoch were included, as to not include undesired edge effects from the Hilbert transform. The final epoch length was therefore $2000 \mathrm{~ms}$, containing phase and amplitude data per $2 \mathrm{~ms}$ for every frequency and channel. Z-values for each subject's EEG amplitude data were obtained in the following way: mean amplitude over the entire epoch was first computed over the trials of each of the three conditions:

Remember trials, if the item was later correctly retrieved with a 'Remember response', Know trials, if the item was later correctly retrieved with a 'Know' response, and Don't Know trials if the item was later responded to with 'Don't know', either correctly or incorrectly.

In order to find significant differences between the conditions, we performed three two-condition comparisons, namely Remember versus Know, Remember versus Don't Know, and Know versus Don't Know. The mean amplitude of each condition's EEG data was then normalized by subtracting the mean amplitude computed over time and over both conditions to be compared, and by dividing by the standard deviation 
computed over time and over both conditions (equation (2); f, c, t are frequency, channel, and time point, respectively, A stands for amplitude, cond 1 and cond 2 stand for the conditions to be compared, and $\mathrm{Z}$ stands for normalized amplitude.). Therefore, if two conditions exhibited the same mean activity, the normalized activity would be zero for both conditions. We adopted this method to compare both the prestimulus and post-stimulus periods directly between two conditions, without resorting to a "baseline". This procedure was repeated for all subjects.

$$
Z_{f, t}=\frac{A_{f, c, t}-\bar{A}_{f, c, \text { cond } 1+\operatorname{con} d 2}}{\sigma_{f, c, \text { cond } 1+\operatorname{con} d 2}}
$$

We statistically compared two conditions with a permutation test (Melloni et al., 2007), by computing the t-statistic with a paired t-test for the normalized amplitude averages per subject and condition, obtained previously. Averages were then shuffled between conditions within and between subjects, and a 'surrogate' t-statistic was obtained for each of 3000 different random shuffles. If the true t-statistic was within the top or bottom $2.5 \%$ of the distribution of 3000 surrogate $t$-statistic values, the difference between conditions was considered significant. In this manner, $p$-values were obtained for all channels, frequencies, and time points, from data used over all participants. Since the comparison between the conditions was made at a great number of channels, we controlled for the multiple comparison problem with the False Discovery Rate (FDR) procedure (Benjamini and Hochberg, 1995).

Since the main results showed significant differences in both the pre- and post-stimulus period, we performed a post-hoc analysis detecting differences between Remember and Know trials in their correlations between prestimulus oscillations with post-stimulus oscillations. Four combinations were assessed: prestimulus "lower theta" (3-5 Hz) correlations with poststimulus "upper theta" (6-8 Hz), and "alpha" (10-12 Hz), and prestimulus upper theta $(6-8 \mathrm{~Hz})$ correlations with poststimulus upper theta $(6-8 \mathrm{~Hz})$ and alpha (10-12 Hz). For each combination, channels showing significant differences in two frequency bands were selected, leading to a set of "pre-channels" (one set for lower theta and one for upper theta) and "post-channels" (one set for upper theta and one for alpha), between which we tested all possible pre-post channel pairs. For each single trial, we obtained the average prestimulus raw amplitude (RA) in lower or upper theta at a pre-channel (-1000 to $-600 \mathrm{~ms})$ and the average RA in upper theta or alpha at a post-channel at each of the following time bins: around onset (-200 to $200 \mathrm{~ms}$ ), early 
poststimulus (200 to $600 \mathrm{~ms}$ ) and late poststimulus (600 to $1000 \mathrm{~ms}$ ).

Pearson's correlation between prestimulus RA and the three poststimulus RAs was computed over trials, participant and condition, resulting in one r-value per channel pair for each participant for both conditions: Remember and Know trials. Between the two groups of r-values, we computed the t-statistic from the paired t-test. We then shuffled the r-values between conditions as described above, obtained a surrogate t-statistic, and repeated this for all 705432 possible combinations. If the true t-statistic lied within the top or bottom $2.5 \%$ of all surrogate t-statistics, the difference in correlation was considered significant. This analysis therefore allowed the identification of a significant difference in pre-post correlation of RA between Remember and Know trials, and was repeated for each channel pair between the prestimulus Region Of Interest (ROI) channel pairs and post-stimulus ROI channel pairs.

All EEG processing steps and statistical analyses were performed with custom-written MATLAB code.

\section{Results}

During the retrieval of the stimuli, mean reaction times for choosing which image was the target image were $8.9076 \mathrm{~s}( \pm 0.1165 \mathrm{~s}, \mathrm{SEM})$ for all correct trials, and $12.6479 \mathrm{~s}( \pm$ $0.2363 \mathrm{~s}$ ) for all incorrect trials. Mean reaction times for the Remember, Know, and Don't Know judgment were $8.5708 \mathrm{~s}$ for Remember trials $( \pm 0.1010 \mathrm{~s}), 9.6088 \mathrm{~s}$ for Know trials ( $\pm 0.1492 \mathrm{~s}$ ), and $12.1106 \mathrm{~s}$ for Don't Know trials ( $\pm 0.2185 \mathrm{~s})$.

The mean duration of the period between the encoding and the retrieval phase, including the arithmetic task, was $22.7093 \mathrm{~s}( \pm 2.0724 \mathrm{~s})$. The time spent on the arithmetic task did not significantly correlate with the number of Remember, Know and Don't Know trials per subject, though there was a slight tendency to positive correlation with the number of Don't Know trials $(r=-0.23, P>0.5$ for Remember; $r=-0.44, P>0.1$ for Know; $\mathrm{r}=0.52, \mathrm{P}=0.0998$ for Don't Know). A one-sided t-test $($ alpha $=0.05)$ was applied, as participants could be expected to perform better than chance but not worse. The t-test revealed that participants selected the correct target image with a probability higher than chance on all trials taken together (Fig. 1B: $78.8 \% \pm 2.2 \%, t(10)=3.47, P<0.005)$, as well as on Know trials $(81.1 \% \pm$ $3.3 \%, t(10)=2.56, P<0.025)$, Remember trials $(96.2 \% \pm 1.6 \%, t(10)=4.32, P<0.001)$, but not on Don't Know trials $(59.6 \% \pm 2.9 \%, t(10)=1.01, P>0.1)$. 
There was a significant interaction between condition and performance (one-way ANOVA, $F(2,30)=49.64, P<<0.0001)$, indicating that participants performed better on Remember trials than on Know trials, and worst on Don't Know trials (Scheffé test, alpha $=0.05$; confidence intervals for Remember vs. Know, $0.1582-0.4432$; Remember vs. Don’t Know, 0.4082 - 0.6932; Know vs. Don't Know, 0.1075 - 0.3925). T-test, ANOVA and Scheffé test results were obtained after performing the arcsine transformation for adapting binomial data to normally distributed data. Since participants accurately identified the target stimulus on Remember and Know trials, but not on Don't Know trials, we used the Don't Know trials as a reference state, to evaluate differences between Remember trials and Know trials as either enhancements or suppressions of oscillations.

We first report EEG results from the prestimulus period (before stimulus onset) in Fig. 2 and 3, then from the post-stimulus period (after stimulus onset) in Fig. 4 and 5.

Finally we elaborate on the connection between prestimulus and post-stimulus activity, with a post-hoc correlation analysis, in Fig. 6.

In order to characterize the differences in EEG activity between Remember and Know trials, we performed a nonparametric statistical permutation test on the amplitudes of oscillations from the encoding phase of Remember trials, Know trials, and Don't Know trials.

The permutation test indicated significant differences between two trial types. Three combinations of two trial types were compared: Remember versus Know trials (Fig. 2A), Remember versus Don't Know trials (Fig. 2C) and Know versus Don't Know trials (Fig. 2D). If we consider Don't Know trials as a reference level for amplitudes, we are able to interpret Remember - Know differences as either enhancements or suppressions of oscillations with respect to Don't Know trials, and therefore as separate characteristics of either the "Remember process" or the "Know process".

We counted the significant points per frequency from all channels over the prestimulus interval of $1000 \mathrm{~ms}$, and corrected this number for frequency-dependent filter tap length, obtaining the total number of cycles that were significantly different between two trial types. This summation allowed us to easily identify the relevant frequencies in the prestimulus period that were dominant in comparing Remember, Know and Don't Know trials (Table 2, Fig. 2A, C-D). 
We found that prestimulus amplitudes in 3-8 Hz were significantly different between Remember trials and Know trials (Table 2; Fig. 2A). The peaks at 4 and $6 \mathrm{~Hz}$ are indicative of theta oscillations. The difference was seen at widespread sites, including frontal, temporal, lateral parietal, and occipital areas (Fig. 2B). Comparing Remember and Don't Know trials also indicates that the Remember process was characterized by enhanced theta oscillations (Fig. 2C). We further observed that both Remember trials and Know trials were characterized by large suppressions in $7-11 \mathrm{~Hz}$, compared to Don't Know trials (Fig. 2C-D; supplementary Fig. 1). Although the 7-11 Hz range overlaps with the theta and alpha oscillations range, the $8 \mathrm{~Hz}$ peak indicates that lower alpha oscillations were most affected. The lower alpha suppression was particularly pronounced in Know trials (Fig. 2D). Raw amplitudes in the prestimulus period, before statistical testing, can be verified in Supplementary Fig. 2.

Two example channels with the different theta amplitude between Remember and Know trials are shown. At the midfrontal channel FC1, a sustained prestimulus difference for Remember and Know trials in $4 \mathrm{~Hz}$ was significant for nearly half of the recorded prestimulus period (Fig. 3A, 3C). Time courses of the mean $4 \mathrm{~Hz}$ raw (non-normalized) amplitude in Remember trials appear to lie below those in Don't Know trials (Fig. 3B), however this difference was not significant at this frequency (Fig. 2B-C).

Left temporal channel T7 exhibited a prestimulus difference in a slightly higher theta oscillation, $6 \mathrm{~Hz}$ (Fig. 3D-F) Note that the difference at this frequency reappeared after stimulus onset (Fig. 3D-F).

In summary, the results from the prestimulus period demonstrate that in Remember trials, prestimulus theta oscillations were enhanced (peaks at 4 and $6 \mathrm{~Hz}$ ). Prestimulus lower alpha oscillations were suppressed in both Remember and Know trials (peak at 8 $\mathrm{Hz})$.

Differences between Remember and Know trials need not be restricted to the prestimulus period right before encoding. Therefore we also analyzed the EEG after stimulus onset (Table 3, Fig. 4). Raw amplitudes can be verified in supplementary Fig. 3. In the post-stimulus period, a significant difference in 6-8 Hz (upper theta) oscillations became apparent between Remember trials and Know trials (Fig. 4A). This difference appeared at left temporal and parietal areas (Fig. 4B). The 6-8 Hz amplitude 
in Remember trials was however only marginally larger compared to Don't Know trials (Fig. 4C, supplementary Fig. 4). In addition to differences in theta oscillations, we also observed that 10-12 Hz (upper alpha) amplitude was higher for Remember trials compared to Know trials, at occipital and frontal sites (Fig. 4A-B) as well as compared to Don't Know trials (Fig. 4C, supplementary Fig. 4). Finally, similarly to the prestimulus period, we again observed strong alpha decrease in Know trials, mostly around $9 \mathrm{~Hz}$ (lower alpha range; Fig. 4D, supplementary Fig. 4).

Highlights of the post-stimulus theta and upper alpha effects for Remember versus Know trials can be seen at temporal channel FT7 (Fig. 5A-C) and frontal channel F8 (Fig. 5D-F) with latencies around $400 \mathrm{~ms}$. Interestingly, prestimulus theta differences appeared at channel F8 before the post-stimulus alpha differences in Remember - Know trials.

To summarize, after stimulus onset, Remember trials showed enhanced theta (peak at 7 $\mathrm{Hz}$ ) and upper alpha (peak at $12 \mathrm{~Hz}$ ) oscillations, at frontal and occipital areas, while Know trials were associated with decreased alpha oscillations, as in the prestimulus period (peak at $9 \mathrm{~Hz}$ ).

In order to find whether the prestimulus theta effects we found were in fact related to the post-stimulus theta and alpha effects, we used a post-hoc correlation analysis based on significant channels and frequencies in our previous results.

We selected all channels with significant differences between Remember trials and Know trials in lower and upper theta frequency bands in the prestimulus period ("pre-channels"), and in upper theta and alpha during the period after onset ("post-channels"), and obtained the average raw amplitude (RA) at these channels during pre- and post-stimulus time bins (Methods). We computed Pearsons' correlation between prestimulus RA at the pre-channels and post-stimulus RA at the post-channels, for each channel pair. Significant differences in correlations were obtained with a t-test and an exhaustive permutation test with t-values (705432 permutations).

Surprisingly, differences in theta-theta correlations were near absent, with only one correlation for Remember trials (Fig. 6A) and one for Know trials (Fig. 6B), appearing earlier.

Theta-alpha correlations however were numerous and widespread during the entire poststimulus period in Know trials (Fig. 6C-D, green arrows), and more restricted for 
Remember trials (orange arrows). Again, Remember was associated with stronger correlations later in the trials (Fig. 6C, rightmost figure). Remember trials only exhibited stronger correlations with prestimulus lower theta (Fig. 6A, C, orange arrows), while Know trials benefited from both low and upper prestimulus theta correlations (Fig. 6A-D, green arrows).

Since these significant differences do not tell us whether the correlation between prestimulus and post-stimulus RA in Know trials and in Remember trials was negative, positive, or zero, we performed a signed rank test (SRT; $v=10$ ) with the r-values for all participants, for each significant pair in Remember trials and in Know trials. Though the t-test results in Fig. 6 indicated many significant differences, the independent evaluations of r-values with the SRT indicated about half of the Remember - Know correlation pairs to not be different from zero (Table 4, rows with n.s.). In total, 9 out of 19 pairs were significant based on the SRT (Table 4, rows with a star; 12 out of 19 pairs if including near significance with $\mathrm{P}<0.06$ ). The significant pairs predominantly represented stronger correlations for Know than for Remember trials (8 out of 9 significant pairs).

In summary, the post-hoc correlation result demonstrates that correlations of prestimulus and post-stimulus theta amplitudes were temporally and spatially distinct for Remember and Know trials. Prestimulus theta to poststimulus alpha correlations were stronger and more numerous for Know trials. Stronger correlations for Know trials were large in number and connected distant areas, while stronger correlations for Remember trials were sparser, more local, uniquely reliant on prestimulus lower theta, and appeared mostly late in the trial.

\section{Discussion}

We aimed to demonstrate whether encoding recognition memory with or without episodic awareness is preceded by different ongoing states of cortical oscillations. Our results are the first to support the existence of this difference, indicating that the encoding of episodic recognition is preceded by a preparatory state, which includes enhancements of theta oscillations in frontal and temporal areas. The enhancements are not present before encoding of non-episodic memory. In encoding episodic memory, theta enhancements reappeared after stimulus onset together with enhancements of upper alpha oscillations. Moreover, non-episodic encoding was characterized by strong 
correlations between prestimulus and poststimulus oscillations over temporal, occipital and frontal areas, while episodic memory exhibited few of such correlations.

Finally, before and during the encoding of both memory types, suppression of lower alpha oscillations was observed.

\section{Theta and pre-post correlation}

Our frequency-specific results indicate that differences between episodic and non-episodic memory are manifested in two theta frequency bands: "lower theta" in 3-5 $\mathrm{Hz}$, and "upper theta" in 6-8 Hz. The two distinct theta peaks are in concordance with a recent study showing the presence of two types of theta in the human hippocampal EEG during the encoding of words (Lega et al., 2012). They demonstrated that lower theta was important for the successful encoding of the words for free recall. Results from the present study suggest that both theta bands are involved in distinguishing successful episodic memory from successful non-episodic memory encoding. During stimulus encoding, increased upper theta marks episodic encoding of the stimulus, and both theta bands are increased in the preparatory state before the stimulus is perceived. Since theta oscillations are enhanced also before retrieval of a stimulus with its source memory (Addante et al., 2011), we propose that ongoing theta activities play a crucial role in qualitative memory processing, and are necessary for encoding as well as retrieval of episodic, contextual memories.

Not only prestimulus activity but also pre- and post-stimulus correlations in the upper theta band differentiate between episodic and non-episodic encoding. Previous works have shown that memory encoding (Osipova et al., 2006), retrieval (Guderian and Düzel, 2005; Anderson et al., 2010), and working memory maintenance (Gevins et al., 1997; Sarnthein et al., 1998; Jensen and Tesche, 2002) are all accompanied by frontal theta activity, and that frontal theta oscillations are coupled to posterior theta (Liebe et al., 2012) and gamma (Friese et al., 2013) during memory tasks.

In our findings, the theta correlation pattern is temporally and spatially distinct for episodic and non-episodic encoding. A previous investigation demonstrated that positive ERPs appear later during the encoding of a stimulus with context, compared to a stimulus without context (Duarte et al., 2004). Our pre-post stimulus correlation results in the theta range are consistent with this; the episodic correlation appeared later than the non-episodic correlation. Moreover, the correlation for episodic encoding 
occupied only the left temporal region, indicating that the left temporal cortex is required both before and after onset for encoding an episode, with its theta frequency increasing from slow to fast. Ongoing theta oscillations may therefore determine later theta activities which control encoding of episodic and non-episodic memory. A causal approach involving active disturbance of theta oscillations is required to ascertain this possibility.

\section{Alpha oscillations and pre-post correlation}

We also demonstrated enhanced post-stimulus upper alpha oscillations in Remember trials that were absent in Know trials. Upper alpha event-related synchronization increases with episodic memory task demands during encoding (Klimesch et al., 1999) and is postulated to reflect working memory operations such as manipulation and maintenance in local sensory modules (Jensen et al., 2002; Kawasaki et al., 2010). Increased prefrontal alpha oscillations reflect "top-down" operations (conscious and intentional cognitive processing) during working memory (Sauseng et al., 2005b). Therefore, top-down processing may be responsible for actively connecting spatiotemporal awareness with the visual stimulus in Remember trials. We observed decreased lower alpha oscillations in both Remember and Know trials, in concordance with decreased alpha oscillations in occipital and frontal regions during successful encoding of meaningful pictures (Friese et al., 2013). The observed suppressions of lower alpha are most probably related to non-stimulus specific, general attentional effects (Klimesch, 1996). Therefore, upper and lower alpha play different roles in memory encoding, with only upper alpha oscillations involved in encoding the episodic nature of the encoded memory. Interestingly, alpha oscillations were not independent of prestimulus theta amplitude, as shown by our post-hoc correlation results. Non-episodic encoding was accompanied by numerous strong correlations between prestimulus theta in the left temporal area and poststimulus alpha in widespread areas. Together with our main results of decreased theta and alpha amplitude in Know trials, this suggests that a decrease in prestimulus theta was indicative of decreased alpha during nonepisodic encoding. The correlation being weak for episodic encoding indicates the different modules in frontal and occipital areas acting more independently, which could allow for encoding a higher degree of information, such as a large number of different episodes of varied nature. 


\section{Ongoing states and default mode network (DMN)}

The present results indicate that ongoing cortical states, besides predicting successful memory formation, determine whether memories are encoded with or without episodic context awareness. A possible functional role of ongoing strong theta activity in Remember trials may be to support a preparatory 'mnemonic context' state of the participant, involving expectations about the task (Guderian et al., 2009).

Task-expectancy related differences in prestimulus theta power have been observed in preparation for a shape or color matching task (Min and Park, 2010). If so, participants should be able to alter their learning context voluntarily. Indeed, anticipatory ERP activity related to an expected reward benefits subsequent memory encoding (Gruber and Otten, 2010).

Another possibility is that the difference in theta states may be related to the ongoing, resting state of the brain known as the default mode network (DMN; Raichle et al., 2001; Fox et al., 2005). Since EEG theta increases are correlated with BOLD deactivation in fMRI (Mizuhara et al., 2004), our results suggest the prestimulus deactivation of midfrontal, temporal and parietal areas, as is the case in the DMN. This network of activations and deactivations relates to internal processing and self-referential thoughts (Gusnard et al., 2001). The "enhanced DMN" apparent by theta frequency oscillation increases in our study may therefore be a preparation to linking subsequent visual stimuli with temporal awareness or existing memories. Further investigation is required to confirm the existence of such a process.

\section{Final discussion and conclusions}

We should point out some limitations of our study. One point is the nature of the Remember/Know task: This task may confound effects on decision confidence (Dunn, 2004; Wixted and Stretch, 2004; Wixted and Mickes, 2010), and is also different from tasks that specifically target source memory (e.g. Summerfield and Mangels, 2005; Addante et al., 2011). Additional tasks are required to elucidate the contribution of confidence strength processes to theta and alpha oscillation changes. Finally, we should emphasize that the findings obtained by correlating prestimulus and post-stimulus theta and alpha oscillations result from a post-hoc analysis, based on relevant frequency ranges and channel sites in our earlier findings. 
In conclusion, our results indicate that non-episodic and episodic memory are determined by qualitatively different ongoing states in the theta range, and that the ongoing state for either memory type is likely to be coordinated with processes during stimulus encoding. Subsequent encoding of episodic memory is supported not only by increased theta oscillations but also upper alpha oscillations, which may be indicative of a difference in working memory processing.

\section{Acknowledgements}

The authors declare no conflict of interest.

The authors thank K. Uchiyama and A. Honda for their help in collecting data, and Y. Uno, M. Vidal-Naquet and K. Yamanaka for helpful discussions.

This work was supported by a MEXT Grant-in-Aid for Scientific Research on Innovative Areas "The study on the neural dynamics for understanding communication in terms of complex hetero systems (No.4103)" (21120005) for FIK, MK, KK, YY, and the JST PRESTO program for KK.

\section{References}

Addante, R.J., Watrous, A.J., Yonelinas, A.P., Ekstrom, A.D., Ranganath, C, 2011. Prestimulus theta activity predicts correct source memory retrieval. Proc. Natl. Acad. Sci. USA 108, 10702-10707.

Anderson, K.L., Rajagovindan, R., Ghacibeh, G.A., Meador, K.J., Ding, M., 2010. Theta oscillations mediate interaction between prefrontal cortex and medial temporal lobe in human memory. Cereb. Cortex 20, 1604-1612.

Babiloni, C., Veccio, F., Mirabella, G., Buttiglione, M., Sebastiano, F., Picardi, A., Di Gennaro, G., Quarato, P.P., Grammaldo, L.G., Buffo, P., Esposito, V., Manfredi, M., Cantore, G., Eusebi, F., 2009. Hippocampal, amygdala, and neocortical synchronization of theta rhythms is related to an immediate recall during Rey auditory verbal learning test. Human Brain Mapp. 30,

2077-2089.

Benjamini ,Y., Hochberg, Y., 1995. Controlling the false discovery rate: a practical and powerful approach to multiple testing. J. R. Statist. Soc. B 57, 289-300. 
Boly, M., Balteau, E., Schnakers, C., Degueldre, C., Moonen, G., Luxen, A., Phillips, C., Peigneux, P., Maquet, P., Laureys, S., 2007. Baseline brain activity fluctuations predict somatosensory perception in humans. Proc. Natl. Acad. Sci. U. S. A. 104, 12187-12192.

Bowles, B., Crupi, C., Mirsattari, S.M., Pigott, S.E., Parrent, A.G., Pruessner, J.C., Yonelinas, A.P., Köhler, S., 2007. Impaired familiarity with preserved recollection after anterior temporal-lobe resection that spares the hippocampus. Proc. Natl. Acad. Sci. USA 104, 16382-16387.

Busch, N.A., Dubois, J., VanRullen, R., 2009. The phase of ongoing EEG predicts visual perception. J. Neurosci. 29, 7869-7876.

Davachi, L., Mitchell, J.P., Wagner, A.D., 2003. Multiple routes to memory: distinct medial temporal lobe processes build item and source memories. Proc. Natl. Acad. Sci. USA 100, $2157-2162$.

Deese, J., Kaufman, R.A., 1957. Serial effects in recall of unorganized and sequentially organized verbal material. J. Exp. Psychol. 54, 180-187.

Delorme, A., Makeig, S., 2004. EEGLAB: an open source toolbox for analysis of single-trial EEG dynamics including independent component analysis. J. Neurosci. Methods 134, 9-21.

De Vanssay-Maigne, A., Noulhiane, M., Devauchelle, A.D., Rodrigo, S., Baudoin-Chial, S., Meder, J.F., Oppenheim, C., Chiron, C., Chassoux, F., 2011. Modulation of encoding and retrieval by recollection and familiarity: Mapping the medial temporal lobe networks.

Neuroimage 58, 1131-1138.

Diana, R.A., Yonelinas, A,P., Ranganath, C., 2007. Imaging recollection and familiarity in the medial temporal lobe: a three-component model. Trends Cogn. Sci. 11, 379-386.

van Dijk, H., Schoffelen, J.M., Oostenveld, R., Jensen, O., 2008. Prestimulus oscillatory 
activity in the alpha band predicts visual discrimination ability. J. Neurosci. 28, 1816-1823.

Duarte, A., Ranganath, C., Winward, L., Hayward, D., Knight, R.T., 2004. Dissociable neural correlates for familiarity and recollection during the encoding and retrieval of pictures. Cogn. Brain Res. 18, 255-272.

Dunn, J.C., 2004. Remember-Know: A matter of confidence. Psychol. Rev. 111, 524-542.

Fell, J., Ludowig, E., Staresina, B.P., Wagner, T., Kranz, T., Elger, C.E., Axmacher, N., 2011. Medial temporal theta/alpha power enhancement precedes successful memory encoding: evidence based on intracranial EEG. J. Neurosci. 31, 5392-5397.

Fox, M.D., Snyder, A.Z., Vincent, J.L., Corbetta, M., Van Essen, D.C., Raichle, M.E., 2005. The human brain is intrinsically organized into dynamic, anti-correlated functional networks. Proc. Natl. Acad. Sci. USA 102, 9673-9678.

Friese, U., Koester, M., Hassler, U., Martens, U., Barreto, N.T., Gruber, T., 2013. Successful memory encoding is associated with increased cross-frequency coupling between frontal theta and posterior gamma oscillations in human scalp-recorded EEG. Neuroimage 66, 642-647.

Gevins, A., Smith, M.E., McEvoy, L., Yu, D., 1997. High-resolution EEG mapping of cortical activation related to working memory: effects of task difficulty, type of processing, and practice. Cereb. Cortex 7, 374-385.

Gruber, M.J., Otten, L.J., 2010. Voluntary control over prestimulus activity related to encoding. J. Neurosci. 30, 9793-9800.

Guderian, S., Düzel, E., 2005. Induced theta oscillations mediate large-scale synchrony with mediotemporal areas during recollection in humans. Hippocampus 15, 901-912. 
Guderian, S., Schott, B.H., Richardson-Klavehn, A., Düzel, E., 2009. Medial temporal theta state before an event predicts episodic encoding success in humans. Proc. Natl. Acad. Sci. USA 106, $5365-5370$.

Gusnard, D.A., Akbudak, E., Shulman, G.L., Raichle, M.E., 2001. Medial prefrontal cortex and self-referential mental activity: relation to a default mode of brain function. Proc. Natl. Acad. Sci., USA 98, 4259-4264.

Hamm, J.P., Dyckman, K.A., McDowell, J.E., Clementz, B.A., 2012. Pre-cue fronto-occipital alpha phase and distributed cortical oscillations predict failures of cognitive control. J. Neurosci. 32, 7034-7041.

Hanslmayr, S., Aslan, A., Staudigl, T., Klimesch, W., Hermann, C.S., Bauml, K., 2007. Prestimulus oscillations predict visual perception performance between and within subjects. Neuroimage 37, 1465-1473.

Hipp, J.F., Engel, A.K., Siegel, M., 2011. Oscillatory synchronization in large-scale cortical networks predicts perception. Neuron 69, 387-96.

Jacoby, L.L., 1991. A process dissociation framework: Separating automatic from intentional uses of memory. J. Mem. Lang. 30, 513-541.

Jensen, O., Gelfand, J., Kounios, J., Lisman, J.E., 2002. Oscillations in the alpha band (9-12 Hz) increase with memory load during retention in a short-term memory task. Cereb. Cortex 12, $877-882$.

Jensen, O., Tesche, C.D., 2002. Frontal theta activity in humans increases with memory load in a working memory task. Eur. J. Neurosci. 15, 1395-1399.

Kawasaki, M., Kitajo, K., Yamaguchi, Y., 2010. Neuronal ensemble for visual working memory via interplay of slow and fast oscillations. Eur. J. Neurosci. 31, 1683-1689. 
Kayser, J., Tenke, C., 2006. Principal components analysis of Laplacian waveforms as a generic method for identifying ERP generator patterns: I. Evaluation with auditory oddball tasks. Clin. Neurophysiol. 117, 348-368.

Kirwan, C.B., Wixted, J.T., Squire, L.R., 2008. Activity in the medial temporal lobe predicts memory strength, whereas activity in the prefrontal cortex predicts recollection. J. Neurosci. 28, 10541-10548.

Klimesch, W., 1996. Memory processes, brain oscillations and EEG synchronization. Int. J. Psychophysiol. 24, 61-100.

Klimesch, W., Doppelmayr, M., Schwaiger, J., Auinger, P., Winkler, Th., 1999. 'Paradoxical' alpha synchronization in a memory task. Cogn. Brain Res. 7, 493-501.

Lega, B.C., Jacobs, J., Kahana, M., 2012. Human hippocampal theta oscillations and the formation of episodic memories. Hippocampus 22, 748-761.

Liebe, S., Hoerzer, G.M., Logothetis, N.K., Rainer, G., 2012. Theta coupling between V4 and prefrontal cortex predicts visual short-term memory performance. Nat. Neurosci. 15, 456-462.

Linkenkaer-Hansen, K., Nikulin, V.V., Palva, S., Ilmoniemi, R.J., Palva, J.M., 2004. Prestimulus oscillations enhance psychophysical performance in humans. J. Neurosci. 24, 10186-10190.

Lisman, J.E., Idiart, M.A., 1995. Storage of 762 short-term memories in oscillatory subcycles. Science 267, 1512-1515.

Mandler, G., 1980. Recognizing: the judgment of previous occurrence. Psychol. Rev. 87, 252-271.

Mathewson, K.E., Gratton, G., Fabiani, M., Beck D.M., Ro, T., 2009. To see or not to see: Prestimulus alpha phase predicts visual awareness. J. Neurosci. 29, 2725-2732. 
Melloni, L., Molina, C., Pena, M., Torres, D., Singer, W., Rodriguez, E., 2007. Synchronization of neural activity across cortical areas correlates with conscious perception. J. Neurosci. 27, 2858-2865.

Min, B., Park, H., 2010. Task- related modulation of anterior theta and posterior alpha EEG reflects top-down preparation. BMC Neuroscience 11,79

Mizuhara, H., Wang, L.Q., Kobayashi, K., Yamaguchi, Y., 2004. A long-range cortical network emerging with theta oscillation in a mental task. NeuroReport 15, 1233-1238.

Osipova, D., Takashima, A., Oostenveld, R., Fernandez, G., Maris, E., Jensen, O., 2006. Theta and gamma oscillations predict encoding and retrieval of declarative memory. J. Neurosci. 26, 7523-7531.

Otten, L.J., Quayle, A.H., Akram, S., Ditewig, T.A., Rugg, M.D., 2006. Brain activity before an event predicts later recollection. Nat. Neurosci. 9, 489-491.

Perrin, F., Pernier, J., Bertrand, O., Echallier, J.F., 1989. Spherical splines for scalp potential and current density mapping. Electroencephalogr. Clin. Neurophysiol. 72, 184-187.

Raghavachari, S., Kahana, M.J., Rizzuto, D.S., Caplan, J.B., Kirschen, M.P., Bourgeois, B., Madsen, J.R., Lisman, J.E., 2001. Gating of human theta oscillations by a working memory task. J. Neurosci. 21, 3175-3183.

Raichle, M.E., Macleod, A.M., Snyder, A.Z., Powers, W.J., Gusnard, D.A., Shulman, G.L., 2001. A default mode of brain function. Proc. Natl. Acad. Sci. USA 98, 676-682.

Rutishauser, U., Ross, I.B., Mamelak, A.M., Schuman, E.M., 2010. Human memory strength is predicted by theta-frequency phase-locking of single neurons. Nature 464, 904-909.

Sarnthein, J., Petsche, H., Rappelsberger, P., Shaw, G.L., Von Stein, A., 1998. 
Synchronization between prefrontal and posterior association cortex during human working memory. Proc. Natl. Acad. Sci. USA 95, 7092-7096.

Sauseng, P., Klimesch, W., Schabus, M., Doppelmayr, M., 2005a. Fronto-parietal EEG

coherence in theta and upper alpha reflect central executive functions of working memory. Int. J. Psychophysiol. 57, 97-103.

Sauseng, P., Klimesch, W., Doppelmayr, M., Percherstorfer, T., Freunberger, R., Hanslmayr, S., 2005b. EEG Alpha synchronization and functional coupling during top-down Processing in a working memory task. Human Brain mapp. 26, 148-155.

Song, Z., Jeneson, A., Squire, L.R., 2011. Medial temporal lobe function and recognition memory: A novel approach to separating the contribution of recollection and familiarity. $J$. Neurosci. 31, 16026-16032.

Summerfield, C., Mangels, J.A., 2005. Coherent theta-band EEG activity predicts item-context binding during encoding. Neuroimage 24, 692-703.

Tesche, C.D., Karhu, J., 2000. Theta oscillations index human hippocampal activation during a working memory task. Proc. Natl. Acad. Sci. USA 97, 919-924.

Tulving, E., 1985. Memory and consciousness. Can. Psychol. 26, 1-12.

Voss, J.L., Paller, K.A., 2009a. Remembering and knowing: electrophysiological distinctions at encoding but not retrieval. Neuroimage 46, 280-289.

Voss, J.L., Paller, K.A., 2009b. An electrophysiological signature of unconscious recognition memory. Nat. Neurosci. 12, 349-355.

Wheeler, M.E., Buckner, R.L., 2004. Functional-anatomic correlates of remembering and knowing. Neuroimage 21, 1337-1349. 
Winson, J., 1978. Loss of hippocampal theta rhythm results in spatial memory deficit in the rat.

Science 201, 160-163.

Wixted, J.T., Stretch, V., 2004. In defense of the signal detection interpretation of remember/know judgments. Psychon. B. Rev. 11, 616-641.

Wixted, J.T., Mickes, L., 2010. A continuous dual-process model of remember/know judgments. Psychol. Rev. 117, 025-1054.

Yonelinas, A.P., Otten, L.J., Shaw, K.N., Rugg, M.D., 2005. Separating the brain regions involved in recollection and familiarity in recognition memory. J. Neurosci. 25, 3002-3008.

Yoo, J.J., Hinds, O., Ofen, N., Thompson, T.W., Whitfield-Gabrieli, S., Triantafyllou, C., Gabrieli, J.D.E., 2012. When the brain is prepared to learn: Enhancing human learning using real-time fMRI. NeuroImage 59, 846-852.

\section{Figure Legends}

Fig. 1. Behavioral task paradigm and behavioral results.

A: Task procedure of one block, with presentation times indicated in ms. Subjects first viewed a sequence of kaleidoscopic images, which were alternated with presentations of a fixation cross ("Encoding” phase). After 12 images, a self-paced arithmetic task was presented twice. Subjects then viewed a short sequence showing two images in succession ("Retrieval" phase), and were prompted to respond with a button press whether the first or the second image shown had appeared in the first part of the block. After responding, subjects were asked to report on their subjective memory by another button press, selecting either "Remember", “Know”, or "Don't Know”. This trial procedure was repeated 10 times, after which a new block was initiated. B: Behavioral results. Left, mean trial numbers for the three trial types. Right, mean performance on trials followed by either a Remember, Know, or Don't Know response. Performance on Remember and Know trials was higher than chance level, as was performance on all trials taken together. Error bars denote the standard error of the mean. 
Fig. 2. Pre-stimulus oscillations in Remember and Know trials. See Table 1 for exact values.

A: Histogram showing the prestimulus amplitude differences between Remember and Know trials in total number of cycles per frequency, summed over all channels.

Oscillations amplitudes were higher in Remember trials compared to Know trials (red bars) in the range 3-8 Hz. Amplitudes that were higher for Know than Remember trials were not found. B: Scalp distributions of differences selected frequency bands from A. The color indicates the total number of significant cycles in the frequency band for any one channel. The frequencies summed are shown below each scalp distribution. C: Histogram with prestimulus amplitude differences between Remember and Don't Know trials. Remember trials showed enhancement in 2 and $4 \mathrm{~Hz}$ (red bars) and suppression of 7-11 Hz oscillations (blue bars) compared to the baseline condition D: Histogram with prestimulus amplitude differences between Know and Don't Know trials. Know trials exhibited a strong suppression of 6-12 Hz compared to the baseline condition (blue bars). Amplitudes that were higher for Know than for Don't Know trials were not found.

Fig. 3. Two examples of channels with prestimulus differences between Remember and Know trials.

A: Topographic distribution of t-values between Remember and Know normalized amplitude in $4 \mathrm{~Hz}$, averaged over the early prestimulus period, and location of channel FC1 (dot). B: Mean time course of $4 \mathrm{~Hz}$ raw amplitude at channel FC1 in Don't Know trials, Know trials and Remember trials. The dashed line represents the time of stimulus onset. C: Time-frequency distribution of t-values between Remember and Know amplitudes at channel FC1, with the significant difference between Remember and Know trials at $4 \mathrm{~Hz}$ (white box). T-values of times and frequencies with p-values below 0.025 , before multiple comparisons correction, are shown in the green box. D:

Topographic distribution of t-values between Remember and Know $6 \mathrm{~Hz}$ amplitude, and location of channel T7. Top, prestimulus period, bottom, post-stimulus (after stimulus onset). E: Mean time course of $7 \mathrm{~Hz}$ raw amplitude at channel $\mathrm{T} 7$ in Don't Know trials, Know trials and Remember trials. F: Time-frequency distribution of t-values between Remember and Know amplitude at channel T7. The significant difference in the 
prestimulus appears in 4 and $6 \mathrm{~Hz}$ (white boxes, left), while the difference in $6 \mathrm{~Hz}$ reappears after stimulus onset (white box, right). All $\mathrm{p}<0.025$ are in the green box.

Fig. 4. Differences in oscillations after stimulus onset.

A: Histogram showing post-onset amplitude differences between Remember and Know trials in total number of cycles per frequency, summed over all channels. We found that 6-8 Hz and 10-12 Hz oscillations were higher in amplitude in Remember trials compared to Know trials (red bars). The post-onset period was 0-1000 ms. Amplitudes that were higher for Know than Remember were not found. B: Scalp distributions of selected frequency bands with significant differences shown in A. C: Histogram with post-stimulus amplitude differences between Remember and Don't Know trials. In Remember trials, 4 and 11-12 Hz (red bars) were enhanced and 7-12 Hz (blue bars) suppressed compared to the baseline condition. D: Histogram with post-stimulus amplitude differences between Know and Don't Know trials. Amplitudes that were higher for Know than Don't Know trials were not found.

Fig. 5. Two examples of channels with post-stimulus onset differences between Remember and Know trials.

A: Topographic distribution of t-values between Remember and Know amplitude in 7 Hz averaged over 0 - $500 \mathrm{~ms}$ after stimulus onset, and location of temporal channel FT7 (dot). B: Mean time course of $7 \mathrm{~Hz}$ raw amplitude at channel FT7 in Don't Know trials, Know trials and Remember trials. C: Time-frequency distribution of t-values between Remember and Know amplitudes at channel FT7, showing the significantly enhanced amplitude in $7 \mathrm{~Hz}$ for Remember trials compared to Know trials after stimulus onset. All $p<0.025$ are in the green box. D: Topographic distribution of mean t-values between Remember and Know amplitude in $12 \mathrm{~Hz}$ after stimulus onset, and location of frontal channel F8. E: mean time course of $12 \mathrm{~Hz}$ raw amplitude at channel F8 in Don't Know trials, Know trials and Remember trials. F: Time-frequency distribution of t-values between Remember and Know amplitude at channel F8, showing the significantly higher amplitude in $12 \mathrm{~Hz}$ for Remember trials compared to Know trials at channel F8 after stimulus onset. A prestimulus difference in $7 \mathrm{~Hz}$ at the same channel is also shown. All $\mathrm{p}<0.025$ are in the green box. 
Fig. 6. Correlation between prestimulus and post-stimulus upper theta amplitude in Remember and Know trials. The colored arrows indicate correlations that were significantly different between Remember trials and Know trials. The gray arrows show the other, n.s. correlations.

A: Pre-post correlation differences for prestimulus lower theta $(3-5 \mathrm{~Hz})$ and poststimulus upper theta $(6-8 \mathrm{~Hz})$. One correlation was stronger in Remember than in Know trials (orange arrow). B: Pre-post correlation differences for prestimulus upper theta and poststimulus upper theta. One early correlation was stronger in Know than in Remember trials (green arrow). C: Pre-post correlation differences for prestimulus low er theta and poststimulus alpha (10-12 Hz). Correlations were consistently more pronounced for Know trials than for Remember trials (green arrows), while a few, mostly local, correlations were higher for Remember trials (orange arrows). D: Pre-post correlation differences for prestimulus upper theta and poststimulus alpha. Three stronger correlations for Know trials were apparent (green arrows). 
Table 1: Remaining number of epochs for analysis after artifact rejection.

\begin{tabular}{|l|l|l|l|}
\hline Participant $\mathrm{nr}$. & Remember trials & Know trials & Don't Know trials \\
\hline 1 & 12 & 30 & 82 \\
\hline 2 & 33 & 51 & 71 \\
\hline 3 & 26 & 27 & 28 \\
\hline 4 & 42 & 63 & 14 \\
\hline 5 & 51 & 40 & 41 \\
\hline 6 & 57 & 61 & 28 \\
\hline 7 & 73 & 39 & 38 \\
\hline 8 & 46 & 14 & 35 \\
\hline 9 & 63 & 46 & 43 \\
\hline 10 & 47 & 46 & 57 \\
\hline 11 & 14 & 42 & 27 \\
\hline
\end{tabular}

Table 2: Number of cycles per frequency in the prestimulus period that are significantly different between trial types, as shown graphically in Fig. 2.

\begin{tabular}{|l|l|l|l|l|l|l|}
\hline & $\begin{array}{l}\text { Total significant } \\
\text { cycles }\end{array}$ & Prestimulus & & & \\
\hline & Remember*Know & $\begin{array}{l}\text { Remember } \\
<\text { Know }\end{array}$ & $\begin{array}{l}\text { Remember } \\
>\quad \text { Don't } \\
\text { Know }\end{array}$ & $\begin{array}{l}\text { Remember } \\
<\quad \text { Don't't } \\
\text { Know }\end{array}$ & $\begin{array}{l}\text { Know > } \\
\text { Don't } \\
\text { Know }\end{array}$ & $\begin{array}{l}\text { Know } \\
\text { Don't } \\
\text { Know }\end{array}$ \\
\hline $2 \mathrm{~Hz}$ & 0.012 & 0 & 0.456 & 0 & 0 & 0 \\
\hline $3 \mathrm{~Hz}$ & 1.3413 & 0 & 0 & 0 & 0 & 0 \\
\hline $4 \mathrm{~Hz}$ & 2.488 & 0 & 0.432 & 0 & 0 & 0.192 \\
\hline $5 \mathrm{~Hz}$ & 0.69 & 0 & 0 & 0 & 0 & 0 \\
\hline $6 \mathrm{~Hz}$ & 2.3855 & 0 & 0 & 0 & 0 & 0.3253 \\
\hline $7 \mathrm{~Hz}$ & 1.2113 & 0 & 0 & 1.7183 & 0 & 19.5775 \\
\hline $8 \mathrm{~Hz}$ & 0.2222 & 0 & 0 & 2.9048 & 0 & 32.0794 \\
\hline $9 \mathrm{~Hz}$ & 0 & 0 & 0 & 2.2679 & 0 & 21.1786 \\
\hline $10 \mathrm{~Hz}$ & 0 & 0 & 0 & 2.1600 & 0 & 16.0800 \\
\hline $11 \mathrm{~Hz}$ & 0 & 0 & 0 & 0.1333 & 0 & 8.3556 \\
\hline $12 \mathrm{~Hz}$ & 0 & 0 & 0 & 0 & 0 & 2.5000 \\
\hline
\end{tabular}


Table 3: Number of cycles per frequency in the post-stimulus period that are significantly different between trial types, as shown graphically in Fig. 4.

\begin{tabular}{|l|l|l|l|l|l|l|}
\hline & $\begin{array}{l}\text { Total significant } \\
\text { cycles }\end{array}$ & Post-stimulus & & & \\
\hline & Remember*Know & $\begin{array}{l}\text { Remember } \\
\text { Know }\end{array}$ & $\begin{array}{l}\text { Remember } \\
>\quad \text { Don't } \\
\text { Know }\end{array}$ & $\begin{array}{l}\text { Remember } \\
<\quad \text { Don't } \\
\text { Know }\end{array}$ & $\begin{array}{l}\text { Know > } \\
\text { Don't } \\
\text { Know }\end{array}$ & $\begin{array}{l}\text { Know } \\
\text { Don't } \\
\text { Know }\end{array}$ \\
\hline $2 \mathrm{~Hz}$ & 0 & 0 & 0 & 0 & 0 & 0.016 \\
\hline $3 \mathrm{~Hz}$ & 0 & 0 & 0 & 0 & 0 & 0 \\
\hline $4 \mathrm{~Hz}$ & 0 & 0 & 0.0640 & 0 & 0 & 0 \\
\hline $5 \mathrm{~Hz}$ & 0 & 0 & 0 & 0 & 0 & 0 \\
\hline $6 \mathrm{~Hz}$ & 1.6627 & 0 & 0 & 0 & 0 & 0.3976 \\
\hline $7 \mathrm{~Hz}$ & 2.5493 & 0 & 0 & 0.4648 & 0 & 1.6620 \\
\hline $8 \mathrm{~Hz}$ & 0.2381 & 0 & 0 & 0 & 0 & 7.5873 \\
\hline $9 \mathrm{~Hz}$ & 0 & 0 & 0 & 0.4643 & 0 & 15.000 \\
\hline $10 \mathrm{~Hz}$ & 0.3800 & 0 & 0.2000 & 0 & 0 & 6.1400 \\
\hline $11 \mathrm{~Hz}$ & 1.5111 & 0 & 0.2444 & 0.6222 & 0 & 1.3333 \\
\hline $12 \mathrm{~Hz}$ & 1.6667 & 0 & 1.0714 & 0.9048 & 0 & 4.2857 \\
\hline
\end{tabular}

Table 4A: Mean r-values over participants and signed rank test results of the pretheta post-theta correlations shown in Fig. 6A-B.

\begin{tabular}{|c|c|c|c|c|c|}
\hline & $\begin{array}{l}\text { Channel } \\
\text { pair }\end{array}$ & Metamemory & Mean $r$ & $\begin{array}{l}\text { Signed } \\
\text { Rank test P }\end{array}$ & $\begin{array}{l}\text { Remember-Know } \\
\text { SRT difference }\end{array}$ \\
\hline \multirow{2}{*}{$\begin{array}{l}\text { Low theta- } \\
\text { high theta }\end{array}$} & & Remember & 0.0982 & 0.2061 & \multirow[t]{2}{*}{ n.s. } \\
\hline & $1 \ldots$ & Know & -0.0649 & 0.5195 & \\
\hline \multirow{2}{*}{$\begin{array}{l}\text { high theta - } \\
\text { high theta }\end{array}$} & \multirow{2}{*}{ 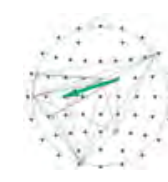 } & Remember & 0.0581 & 0.1016 & \multirow[t]{2}{*}{$*$} \\
\hline & & Know & 0.2176 & 0.0098 & \\
\hline
\end{tabular}


Table 4B: Mean r-values over participants and signed rank test results of the pretheta post-al pha correlations shown in Fig. 6C-D.

\begin{tabular}{|c|c|c|c|c|c|}
\hline & $\begin{array}{l}\text { Channel } \\
\text { pair }\end{array}$ & Metamemory & Mean $r$ & $\begin{array}{l}\text { Signed } \\
\text { Rank test P }\end{array}$ & $\begin{array}{l}\text { Remember-Know } \\
\text { SRT difference }\end{array}$ \\
\hline \multirow{10}{*}{$\begin{array}{l}\text { Low } \\
\text { theta-alpha } \\
\text { (onset) }\end{array}$} & & Remember & 0.105 & 0.2061 & \multirow[t]{2}{*}{ n.s. } \\
\hline & & Know & -0.0552 & 0.4648 & \\
\hline & & Remember & -0.0095 & 0.4648 & \multirow[t]{2}{*}{$*$} \\
\hline & 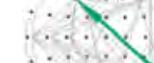 & Know & 0.1507 & 0.0244 & \\
\hline & & Remember & -0.0117 & 0.8984 & \multirow[t]{2}{*}{ * } \\
\hline & & Know & 0.1072 & 0.0244 & \\
\hline & & Remember & -0.1311 & 0.1016 & \multirow[t]{2}{*}{ n.s. } \\
\hline & & Know & 0.0552 & 0.2061 & \\
\hline & & Remember & -0.0472 & 0.2402 & \multirow[t]{2}{*}{$*$} \\
\hline & & Know & 0.1053 & 0.0049 & \\
\hline \multirow{7}{*}{$\begin{array}{l}\text { Low } \\
\text { theta-alpha } \\
\text { (early post) }\end{array}$} & & Remember & -0.0606 & 0.3203 & \multirow[t]{2}{*}{ n.s. } \\
\hline & 8 & Know & 0.0294 & 0.5195 & \\
\hline & & Remember & -0.0926 & 0.0674 & \multirow[t]{2}{*}{$*$} \\
\hline & & Know & 0.0927 & 0.0322 & \\
\hline & & Remember & -0.0755 & 0.0186 & \multirow[t]{2}{*}{$*$} \\
\hline & & Know & 0.1183 & 0.0537 & \\
\hline & & Remember & -0.0967 & 0.2061 & n.s. \\
\hline
\end{tabular}




\begin{tabular}{|c|c|c|c|c|c|}
\hline & & Know & 0.0419 & 0.5771 & \\
\hline & \multirow{2}{*}{$\frac{1}{\because:}$} & Remember & -0.0369 & 0.4131 & \multirow[t]{2}{*}{$*$} \\
\hline & & Know & 0.1700 & 0.0420 & \\
\hline \multirow{8}{*}{$\begin{array}{l}\text { Low } \\
\text { theta-alpha } \\
\text { (late post) }\end{array}$} & & Remember & 0.1649 & 0.0537 & n.s. \\
\hline & & Know & -0.0221 & 0.7002 & \\
\hline & & Remember & -0.0282 & 0.3203 & $*$ \\
\hline & & Know & 0.1396 & 0.0137 & \\
\hline & & Remember & 0.0019 & 0.9658 & ns. \\
\hline & & Know & 0.1197 & 0.0537 & \\
\hline & & Remember & 0.0637 & 0.3652 & $*$ \\
\hline & & Know & -0.0833 & 0.0830 & \\
\hline High & & Remember & -0.0059 & 1 & n.s. \\
\hline $\begin{array}{l}\text { theta-al pha } \\
\text { (onset) }\end{array}$ & $\because$ & Know & 0.1241 & 0.0537 & \\
\hline High & & Remember & -0.1064 & 0.1475 & n.s. \\
\hline theta-alpha & & Know & 0.0017 & 1 & \\
\hline & & Remember & -0.0679 & 0.2061 & n.s. \\
\hline & & Know & 0.0489 & 0.5195 & \\
\hline
\end{tabular}




\section{Figure 1}
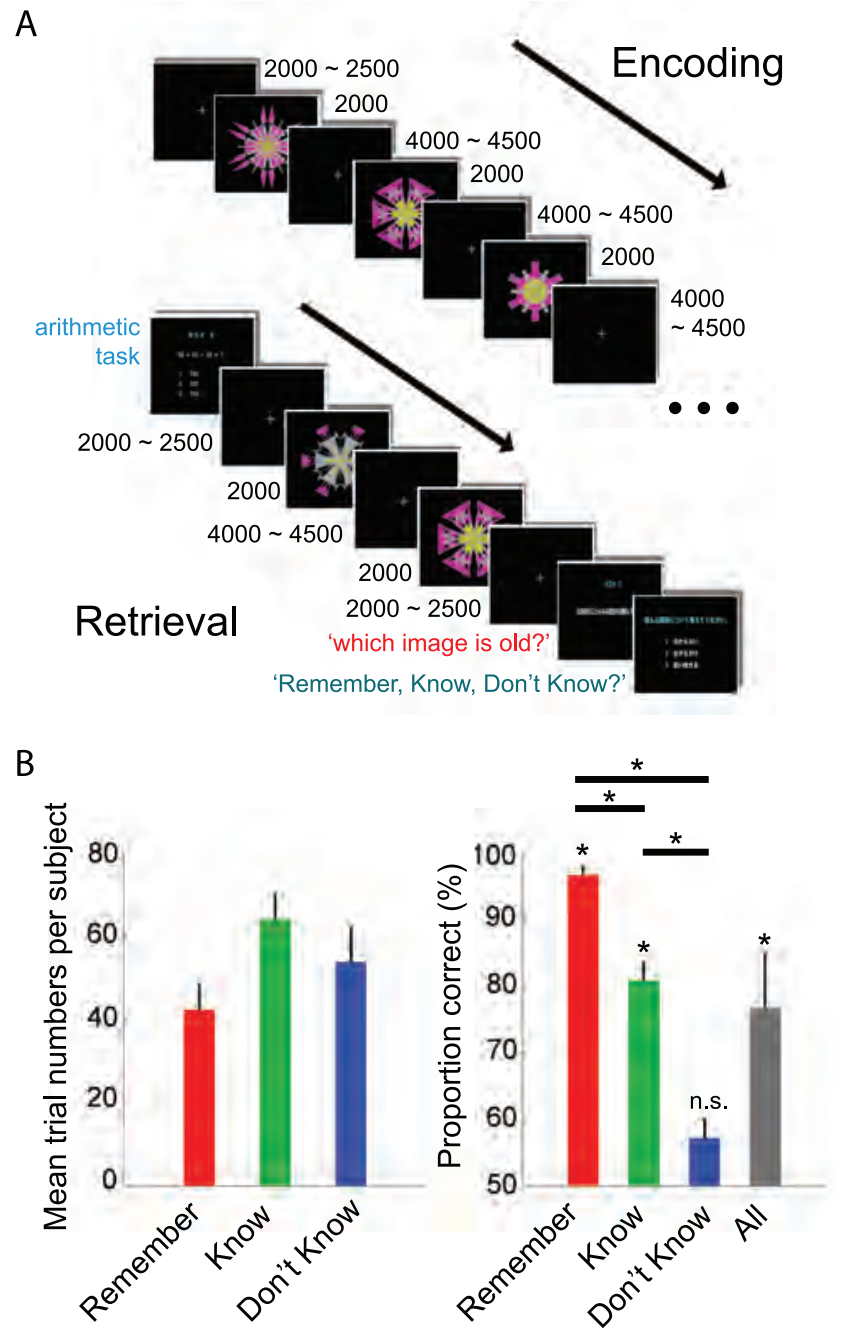
Figure 2

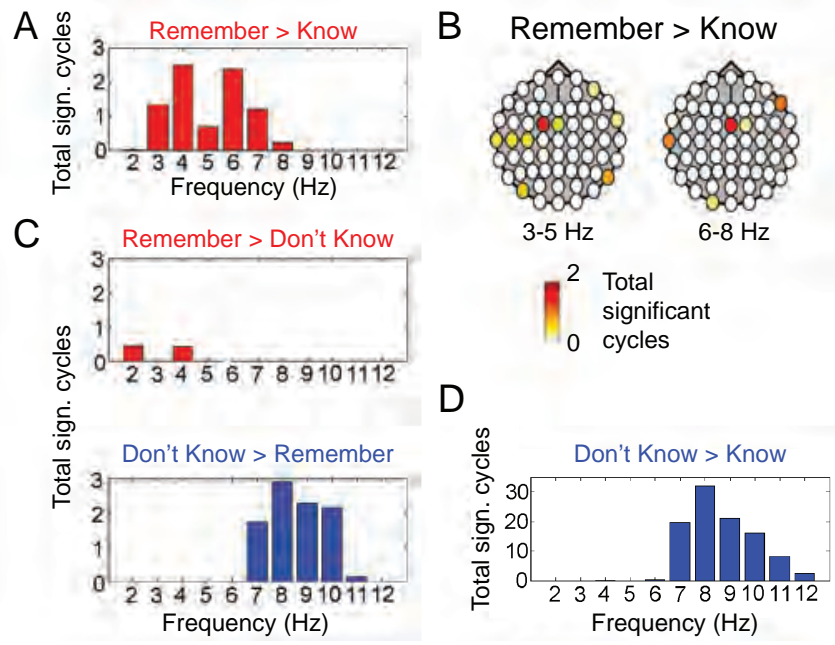




\section{Figure 3}

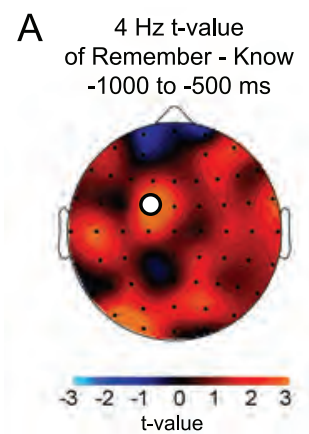

D

$6 \mathrm{~Hz}$ t-value of Remember - Know

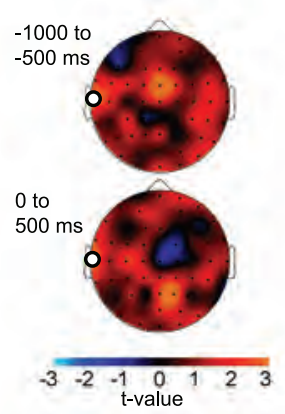

B $4 \mathrm{~Hz}$ mean raw amplitude timecourse at channel FC1

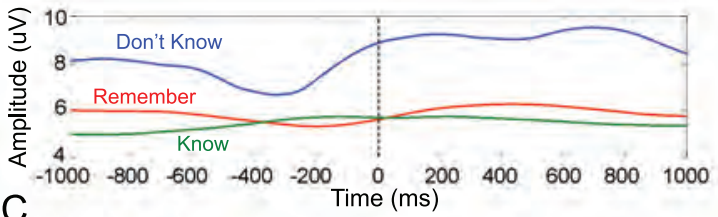

C

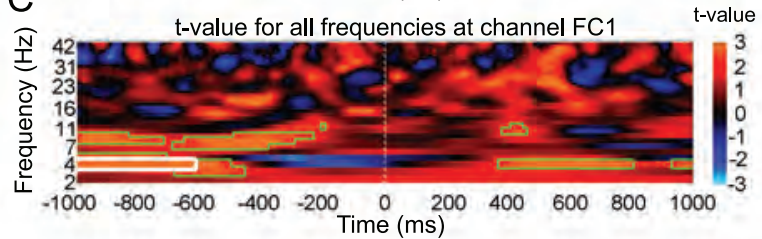

E $6 \mathrm{~Hz}$ mean raw amplitude timecourse at channel T7

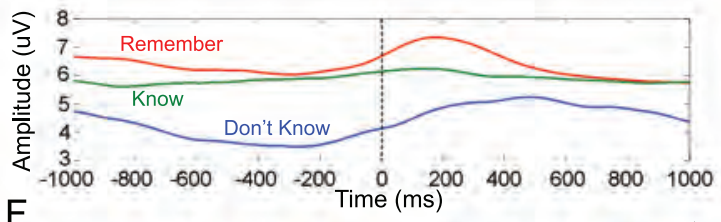

F

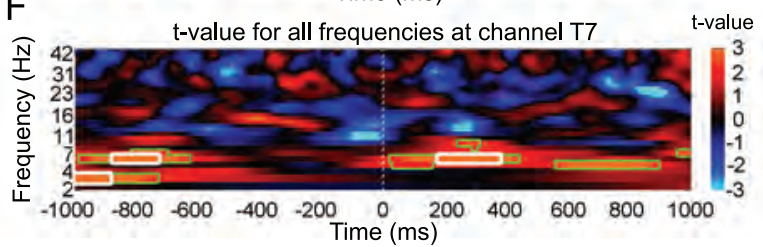


Figure 4

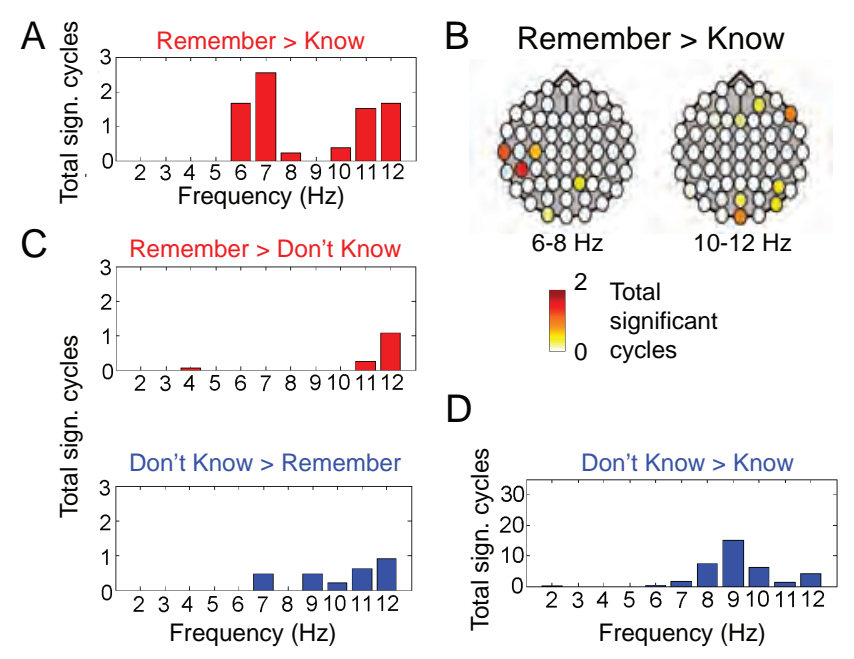




\section{Figure 5}

A $\quad 7 \mathrm{~Hz}$ t-value of Remember - Know 0 to $500 \mathrm{~ms}$
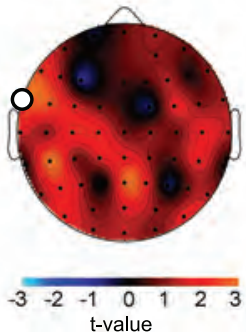

t-value

D $12 \mathrm{~Hz}$ t-value of Remember - Know 400 to $600 \mathrm{~ms}$

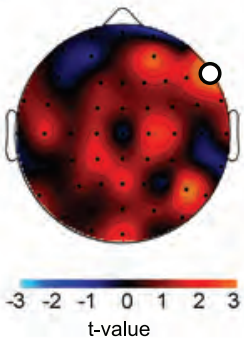

B

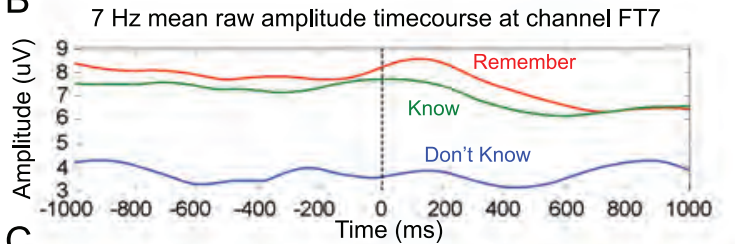

C

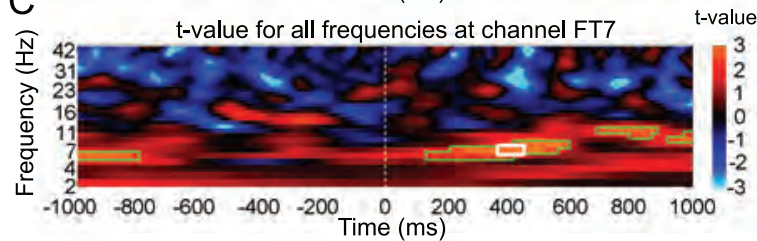

E $\quad 12 \mathrm{~Hz}$ mean raw amplitude timecourse at channel F8

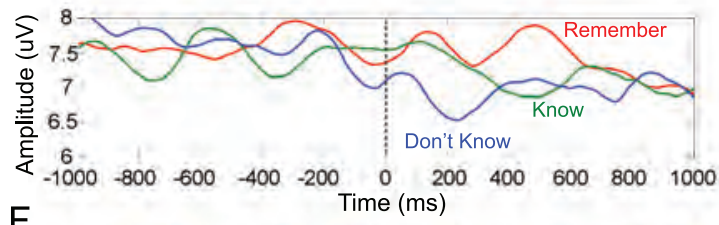

$\mathrm{F}$

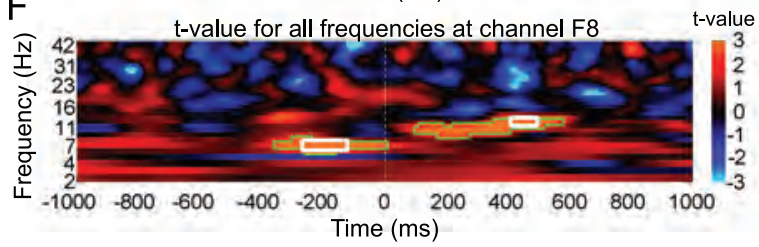




\section{Figure 6}

Pre-Post correlations in theta and alpha amplitude

around
onset
$-200 \sim 200 \mathrm{~ms}$

A

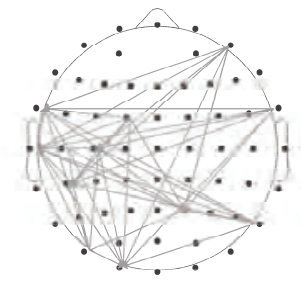

B

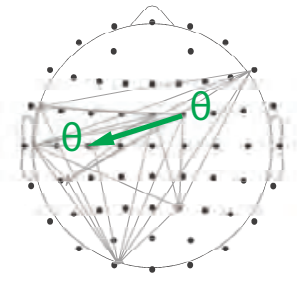

C

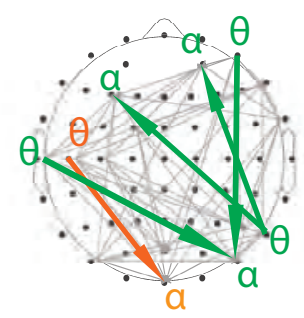

D

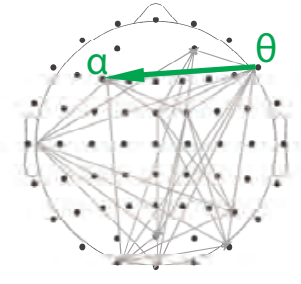

$$
\begin{gathered}
\text { early } \\
\text { postsimulus } \\
200 \sim 600 \mathrm{~ms}
\end{gathered}
$$

low theta $\rightarrow$ high theta

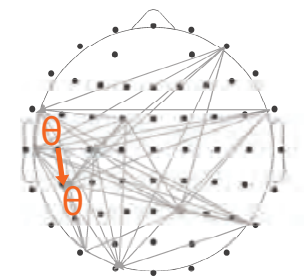

high theta $\rightarrow$ high theta

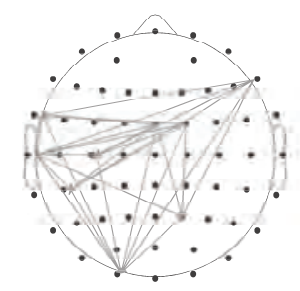

low theta $\rightarrow$ alpha

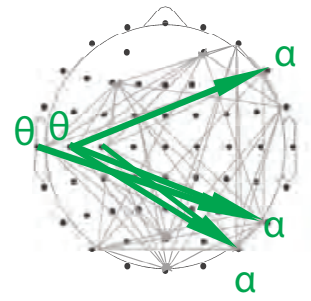

high theta $\rightarrow$ alpha
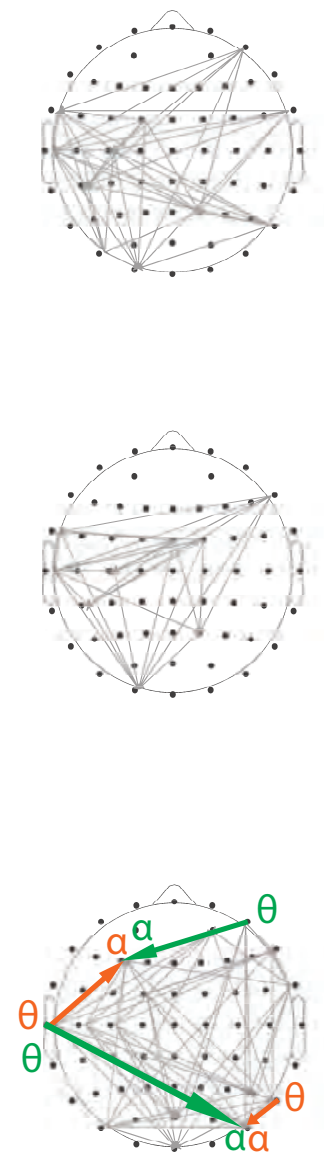
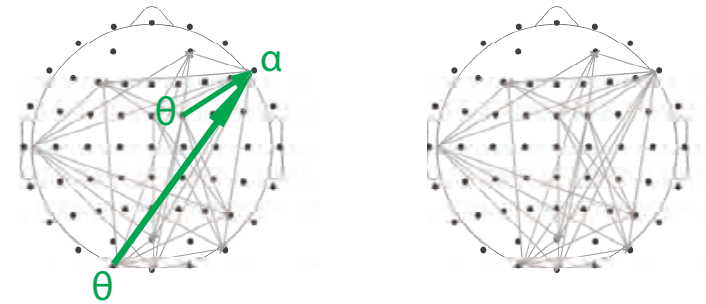